\title{
The central regulator p62 between ubiquitin proteasome system and autophagy and its role in the mitophagy and Parkinson's disease
}

\author{
Woo Hyun Shin, Joon Hyung Park \& Kwang Chul Chung* \\ Department of Systems Biology, College of Life Science and Biotechnology, Yonsei University, Seoul 03722, Korea
}

\begin{abstract}
The ubiquitin-proteasome system (UPS) and autophagy are two major degradative pathways of proteins in eukaryotic cells. As about $30 \%$ of newly synthesized proteins are known to be misfolded under normal cell conditions, the precise and timely operation of the UPS and autophagy to remove them as well as their tightly controlled regulation, is so important for proper cell function and survival. In the UPS, target proteins are labeled by small proteins called ubiquitin, which are then transported to the proteasome complex for degradation. Altematively, many greatly damaged proteins are believed to be delivered to the lysosome for autophagic degradation. Although these autophagy and UPS pathways have not been considered to be directly related, many recent studies proposed their close link and dynamic interconversion. In this review, we'll focus on the several regulatory molecules that function in both UPS and autophagy and their crosstalk. Among the proposed multiple modulators, we will take a closer look at the so-called main connector of UPS-autophagy regulation, p62. Last, the functional role of p62 in the mitophagy and its implication for the pathogenesis of Parkinson's disease, one of the major neurodegenerative diseases, will be briefly reviewed. [BMB Reports 2020; 53(1): 56-63]
\end{abstract}

\section{INTRODUCTION}

The ubiquitin-proteasome system (UPS) and autophagy are two major degradative pathways in eukaryotic cells (1). They are considered to act in a complementary manner. Although small misfolded and damaged proteins are generally modified by ubiquitin and destined for the proteasomal degradation, many large and damaged proteins are delivered to the lysosome for

*Corresponding author. Tel: +82-2-2123-2653; Fax: +82-2-3125657; E-mail: kchung@yonsei.ac.kr

https://doi.org/10.5483/BMBRep.2020.53.1.283

Received 14 October 2019

Keywords: Autophagy, Mitophagy, p62, Parkinson's disease, Protein quality control, Ubiquitination, Ubiquitin Proteasome System autophagic degradation (2). Protein ubiquitination is a kind of post-translational modification and plays a central role in both degradative pathways. Ubiquitination of target proteins acts as a signal either for the substrates to be degraded by proteasome or lysosome or as an indicator for modulating their non-proteolytic processes (3). Ubiquitin is a 76-amino-acid long protein, and a single or a chain of ubiquitin can be conjugated mainly to lysine residues on the protein substrate via an isopeptide bond. Secondary ubiquitin molecules are linked to one of the seven lysine residues (K6, K11, K27, K29, $\mathrm{K} 33, \mathrm{~K} 48$, and $\mathrm{K} 63$ ) or to the $\mathrm{N}$-terminal methionine of the previous ubiquitin molecule $(4,5)$. In the UPS, polyubiquitin chains determine the degradation mode. With K48-linked chains, the protein target tends to be degraded by the UPS, and the K63-linked chain or monoubiquitinated substrates are modified by autophagy (6).

Like the UPS, autophagy is also essential for accurate cellular and energy homeostasis. There are three main types of autophagy, namely, macroautophagy, microautophagy and chaperone-mediated autophagy (2). They are mediated by the autophagy-related genes and their associated enzymes (2). Among them, macroautophagy is then divided into bulk and selective autophagy, which proceeds in several stages: initiation, nucleation, elongation, maturation, and fusion with the lysosome (7). Autophagy can be triggered by various adverse conditions, such as nutrient starvation, infection, oxidative stress, protein aggregation, or the inhibition of the mTOR pathway. As well, formation of the protein complex including Unc-51-like autophagy activation kinase 1 (ULK1), RB1-inducible coiled-coil 1 (RB1CC1; also known as FIP200), and the autophagy-related gene 101 (ATG101) protein induces the autophagy (7). The next nucleation step, including the formation of phagophore, starts with the release of Beclin1 and AMBRA1 from Bcl-2. The participation and activation of phosphoinositide 3-kinase, vacuolar protein sorting 34 (VPS34), multiple ATG products, and VPS15 then follows in the nucleation of autophagic vesicles (8).

In the elongation stage many ubiquitin-like conjugation moieties are produced and facilitate the formation of the macromolecular complexes. This step is initiated by the combined action of ATG7 (having the ubiquitin-activating E1

ISSN: 1976-670X (electronic edition)

Copyright (C) 2020 by the The Korean Society for Biochemistry and Molecular Biology

(ㄷ) This is an open-access article distributed under the terms of the Creative Commons Attribution Non-Commercial License (http://creativecommons.org/licenses/by-nc/4.0) which permits unrestricted non-commercial use, distribution, and reproduction in any medium, provided the original work is properly cited. 
enzyme activity), which then activates the ubiquitin-like protein ATG12. The ATG7-ATG12 complex is then transferred to ATG10 (acting as the ubiquitin-conjugating E2 enzyme-like) and is covalently linked to ATG5. The ATG5-ATG12 complex is ultimately conjugated with ATG16 for the elongation of the phagophore (9). Next, the microtubule-associated protein 1A/B light chain 3B (LC3) is conjugated to phosphatidylethanolamine, which is mediated by ATG3 and ATG7. LC3 remains attached to the lipid, while ATGs are dissociated after the autophagosome formation (10). After being transported along with microtubules, soluble NSF attachment receptor (SNARE)-like proteins, including STX17, SNAP29, and VAMP8, stimulates the fusion of autophagosomes and lysosomes into autophagolysosomes (11).

Recent studies have suggested the possibility of crosstalk between the UPS and autophagy. Several molecules are shared either as the regulators or common substrates of the UPS and autophagy pathways (12). One well-known regulator that marks the target for degradation via both pathways is the ubiquitin (13). In the UPS, the target protein is conjugated with a polyubiquitin chain destined for proteasomal degradation. In autophagy, a polyubiquitin chain is also recognized by the autophagy adaptor protein, p62, for fusion and subsequent targeting into the autophagosomes (14). Many protein factors have now been shown to regulate these two intracellular proteolytic pathways (Table 1).

\section{THE UPS AND AUTOPHAGY ARE TIGHTLY ASSOCIATED}

In the UPS, the ubiquitin chain acts as a signal that promotes the polyubiquitination of the target protein, enabling its access to the proteasome, where it is degraded and recycled as amino acids (15). Deubiquitinating enzymes (DUBs) play vital roles in the UPS by removing ubiquitin from substrate proteins and regulating their proteasomal degradation as well as sublocalization (16). On the other hand, autophagy is a cellular response and becomes activated when cells are subjected to serum starvation. Autophagy pathway promotes a vesicular trafficking to the lysosome (17). To date, the UPS and autophagy have been considered mutually irrelevant (18). However, there are several reports that autophagy is activated when the UPS is dysregulated. In addition, several proteins, such as USP14, El24, p62, and HDAC6, have been proposed as regulating both autophagy and the UPS (19).

Ubiquitin-specific protease 14 (USP14), which belongs to one of the five subgroups of DUBs, deubiquitinates the ubiquitin moiety from the target protein at the K48 residue and inhibits the UPS. In addition, USP14 negatively controls the K63-linked ubiquitination of Beclin1 to regulate the autophagy. When Akt phosphorylates USP14 at Ser432, it augments both K48- and K63-linked deubiquitination activity of USP14, affecting autophagy negatively. Another study suggests that Akt-regulated USP14 activity modulates both the UPS and autophagy (20). USP14 can interrupt the association between TRAF6 and Beclin1 to suppress autophagy. Upon TLR4 stimulation, TRAF6 interacts with Beclin1 to induce the ubiquitination of Beclin1, which leads to the activation of the autophagic pathway. In this condition, USP14 competitively interacts with Beclin1, which then impedes the ubiquitination of Beclin1 by TRAF6 (21). Moreover, USP14 is required for the fusion of autophagosomes. When USP14 activity is inhibited, intracellular proteasome activity is correspondingly increased (22), whereas the autophagy flux is impeded at the step of autophagosome-lysosome fusion (22). Furthermore, treatment of cultured cells with a small chemical inhibitor of USP14, IU1, considerably inhibits the oligomerization of microtubuleassociated protein tau protein (22). In contrast, the blockage of USP14 activity significantly upregulates the level of huntingtin with 97-polyglutamine repeats (22). These diverse outcomes imply that the effect of USP14 inhibition largely depends on the selective cellular context and appears to be substratespecific.

Etoposide-induced protein 2.4 homolog (EI24) was first identified as a tumor suppressor gene and its expression is primarily regulated by p53 (23). The El24 protein prominently reduces cell growth in COS-7 cells (23). El24 also inhibits cell growth and promotes apoptosis in several cancer cells (24).

Table 1. List of currently known and key modulators to regulate and fine-tune both the UPS and autophagy-mediated proteolytic processes

\begin{tabular}{|c|c|c|c|}
\hline Gene & Reported cellular function & Regulatory role between UPS and autophagy & References \\
\hline USP14 & $\begin{array}{l}\text { Deubiquitinates K48 for downregulating } \\
\text { ubiquitin-proteasome system }\end{array}$ & $\begin{array}{l}\text { Modulates autophagy through } \mathrm{K} 63 \\
\text { deubiquitination }\end{array}$ & Xu D et al. (2016) \\
\hline El24 & $\begin{array}{l}\text { Suppresses cell growth and induces apoptosis, acts } \\
\text { as tumor suppressor }\end{array}$ & $\begin{array}{l}\text { Induces autophagy-dependent degradation of } \\
\text { RING E3 ligases }\end{array}$ & $\begin{array}{l}\text { Zhao YG et al. (2012) } \\
\text { Devkota S et al. (2016) }\end{array}$ \\
\hline p62 & $\begin{array}{l}\text { Acts as a signaling mediator that located in late } \\
\text { endosome and lysosome }\end{array}$ & $\begin{array}{l}\text { Targets ubiquitinated proteins to the } \\
\text { autophagosome entry }\end{array}$ & $\begin{array}{l}\text { Hou B et al. (2019) } \\
\text { Matsumoto G et al. (2011) }\end{array}$ \\
\hline ATG16 & $\begin{array}{l}\text { Localized in the cytosol and plays role in } \\
\text { autophagosome formation }\end{array}$ & $\begin{array}{l}\text { Binds with proteasome component PSMD1 or } \\
\text { PSMD2 for degradation in lysosome }\end{array}$ & $\begin{array}{l}\text { Kuma A et al. (2002) } \\
\text { Xiong Q et al. (2018) }\end{array}$ \\
\hline HDAC6 & $\begin{array}{l}\text { Promotes autophagy and regulates fusion } \\
\text { autophagosome to lysosome }\end{array}$ & $\begin{array}{l}\text { Targets ubiquitinated proteins and leads to form } \\
\text { aggresome for autophagic degradation }\end{array}$ & $\begin{array}{l}\text { Lee et al. (2010) } \\
\text { Kawaguchi Y et al. (2003) }\end{array}$ \\
\hline
\end{tabular}


However, a few studies have reported that El24 is an essential component of macroautophagy, acting as a tumor promoter (24). This role is contrary to those from many previous reports. Involvement of El24 in autophagy was first reported in Caenorhabditis elegans (25), followed by the finding that it is also an essential component of autophagy in mammals (26). For example, knockdown of El24 by using siRNA in pancreatic tumor cells impaired autophagy, causing the inhibition of cell proliferation. However, this effect was not seen in other cell lines (24). Overexpression of El24 attenuated NK-кB activity by interacting with TRAF2. Furthermore, the RING domain of TRAF2 is an essential part of its binding to El24 and subsequent autophagy-dependent protein degradation (27).

El24 also binds to E3 ubiquitin ligase TRIM41 to be degraded, but its detailed mechanism was not well elucidated (28). Whereas TRIM41 is usually autoubiquitinated and undergoes proteasome-dependent degradation (29), EI24 overexpression reduced the level of ubiquitinated TRIM41, and induced autophagy-dependent degradation. To examine whether the RING domain of TRIM41 is required for El24-mediated auto-degradation, several proteins either including the RING domain or not were screened for their binding to El24 and subsequent proteolysis. The results revealed that some proteins were degraded (TRAF6, BIRC2/CIAP1, and MDM2), but not others (PARK2, XIAP, and BIRC3/CIAP2), irrespective of the presence of the RING domain (30). These results indicated that El24-mediated autophagy and subsequent degradation may affect the E3 ligases activity via the RING domain but the RING domain is not the only requirement for El24-mediated degradation (30).

It was originally found that p62/SQSTM1 (or referred to as sequestosome 1) is a signaling mediator and is located in late endosome and lysosome (31). It can act as a multifunctional adaptor protein that regulates numerous cellular functions, such as nutrient sensing and apoptotic modulation (32). Several recent studies revealed the new functional role of p62. Although autophagic protein degradation was thought to be a compensatory mechanism of the UPS, p62 can link these two cellular degradation pathways. The p62 protein is selectively degraded by autophagy, whereas it can also act as the cargo receptors or adaptors for the autophagic degradation of ubiquitinated substrate proteins (33). When p62 is phosphorylated at the Ser403 residue in the UBA domain, it stabilizes the structure of sequestosome as a cargo of ubiquitinated proteins for the entry of autophagosomes. However, p62 itself was not degraded by the proteasome (34).

The core autophagy protein ATG16 also appears to play a modulatory role between the UPS and autophagy. ATG16 was known to be an essential component of the ATG complex, and the ATG12-ATG5 complex binds to ATG16 for the phagophore formation (35). ATG16 is thought to be regulated possibly by the UPS, based on the finding that treatment of MEFs with proteasome inhibitor led to an increase of ATG16 (36). Moreover, ATG16 seems to be crucial for the function of the
UPS in the Dictyostelium discoideum, because the proteasome activity was remarkably down-regulated in the ATG16-KO mutant of Dictyostelium (37). Interestingly, PSMD1 and PSMD2, the components of the 195 regulatory particle in the proteasome complex, directly interact with ATG16. When ATG16 is bound to PSMD1 or 2, it is degraded by the lysosomes, and ATG16 was an essential component of autophagy progression (38)

The histone deacetylase-6 (HDAC6) has emerged as an important player in the cellular management of protein aggregates (39). It acts as the major regulatory component of autophagy-dependent clearance of misfolded-proteins. HDAC6 directly interacts with polyubiquitinated proteins and binds to dynein motor proteins for the recruitment of the misfolded proteins to the aggresome (40). In addition, HDAC6 recognizes the ubiquitinated protein aggregates and transports the cargo to the microtubule organizing center to form aggresomes for autophagic degradation $(39,40)$. HDAC6 also functions together with p62/SQSTM1 in the mitophagy $(41,42)$. In addition, a recent study unveiled the autophagy regulation function of HDAC6. A UPS-impaired fly model displayed the compensatory activation of the autophagic activity in an HDAC6-dependent manner. Furthermore, when the fly model of neurodegeneration generated by proteasome impairment was overexpressed with HDAC6, the decrease of cell viability was rescued in an autophagy-dependent manner (43). These results verified the dual-regulatory role of HDAC6 between the UPS and autophagy.

\section{THE MAIN CONNECTOR BETWEEN THE UPS AND AUTOPHAGY, p62, ALSO REGULATES THEIR CROSSTALK}

About $30 \%$ of newly synthesized proteins are known to be misfolded in normal cell conditions (44). Because these misfolded proteins form aggregates and interfere with the normal cell function (45), they must be removed quickly and accurately. In such a protein-quality control, both the UPS and autophagy and their precise control play a crucial role (39, 46). As described previously, the UPS and autophagy can affect each other rather than operate independently, and there are several control proteins that work together on both pathways in a cooperative and complementary manner.

Several studies have demonstrated that autophagy is activated when the proteasome could not properly remove the polyubiquitinated target proteins (47-49). Inhibition of the intracellular proteasome complex with diverse types of inhibitors were found to induce the compensatory autophagic pathway. The activation of the autophagy pathway can be largely divided into the process of compensatory activation in cytosol to remove the ubiquitinated proteins, as well as the process of proteasomal inhibition in ER, sequentially resulting in ER stress and unfolded protein response, and the induction of multiple ATG genes (50). On the other hand, the inhibition 
of autophagy can impair the UPS $(34,51,52)$. Many studies have suggested that this difference results from the novel regulatory function of p62 between these two systems.

It is well known that p62 is a multi-functional protein that plays an important role in autophagy $(14,53,54)$. It consists of a variety of domains that can bind to different proteins (55). Several domains, including the UBA, LIR, and PB1 domains, allow the binding of p62 to the ubiquitin, LC3, and many other proteins (56). It also has the signal sequence domain of nuclear localization (NLS) and nuclear export (NES), so it can rapidly shuttle between the nucleus and cytosol (32). In particular, the PB1 domain allows p62 to directly bind to the proteasome complexes. It also allows the p62 to form oligomers with other PB1 domain-containing proteins. The p62 level is regulated by means of several pathways, such as the signal transduction pathways activated by starvation or oxidative stress, the Ras/MAPK pathway, and the JNK/c-Jun pathway (57). Proteasome inhibition is also known to increase the p62 level (58). Treatment of proteasome inhibitors, such as epoxomicin and MG132, increases the p62 transcription (59). Because the p62 is a proteolytic substrate during the autophagic process, the amount of intracellular p62 also decreases as the extent of the autophagy reaction is increased (60).

p62 was initially known as an adapter protein of autophagy $(14,53)$. However, further studies have shown that p62 can bind to the Rpt 1 and Rpt10 of the 265 proteasome complex

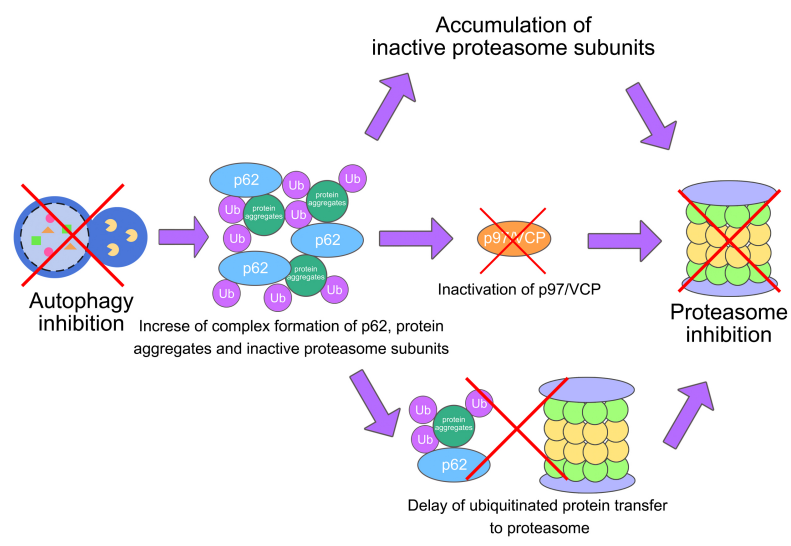

Fig. 1. The inhibition of autophagy results in the inactivation of UPS. Autophagy inhibition causes the accumulation of the p62 complex, including protein aggregates, resulting in the delayed transport of ubiquitinated protein substrates to proteasomes. Moreover, the abnormal protein aggregates with p62 can inactivate the multiple regulators of the UPS, such as p97/VCP. The p62aggregates also contained inactive components of the $26 \mathrm{~S}$ proteasome complex as well as ubiquitinated proteins and the autophagosome. Accumulation of those inactive proteasome components would suppress the removal of ubiquitinated target proteins through the UPS. Thus, the inhibition of autophagy consequently reduces the proteasome degradation of ubiquitinated targets through various p62-regulated pathways. through the PB1 domain $(59,61)$. It also binds to the ubiquitinated protein via the UBA domain, and then drags them toward the proteasome, which in turn promotes their degradation via the UPS. This action occurs not only in the cytosol but also in the nucleus. In this process, p62 uses the NLS and NES to shuttle between the cytosol and the nucleus, and carry the target protein from the nucleus to the proteasome in the cytosol $(32,62,63)$. In addition to unmodified protein, p62 can recruit and transport the ubiquitinated proteins from nucleus to cytosol during this process $(62$, Fig. 1$)$.

Long-term inhibition of autophagy reduces the proteasomal degradation of ubiquitinated proteins, which involve p62 (52). Autophagy inhibition causes accumulation of the complex consisting of p62 and protein aggregates, which results in the delayed migration of ubiquitinated substrates to the proteasomes (52). In addition to the failure of transport of proper ubiquitinated protein targets and so resultant positional isolation, abnormal p62 aggregates can also functionally inactivate the regulators of the UPS, such as the p97/VCP (64). In this process, the binding between the PB1 domain and UBA domain of p62 and the proteasome regulator is critically required.

The p62 aggregates also contain inactive proteasome components as well as the ubiquitinated proteins and autophagosome (59). Many recent studies have shown that inactivated proteasome components are ubiquitinated and migrate to the autophagosomes for the lysosomal degradation under cellular stress, such as nutrient starvation $(65,66)$. Aged

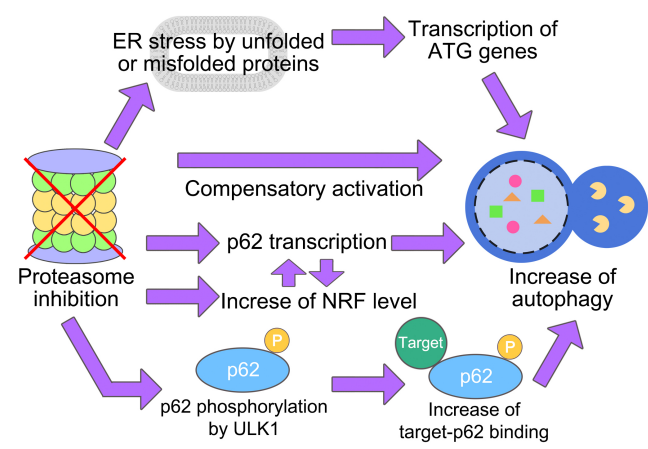

Fig. 2. The inhibition of the UPS results in the activation of autophagy. Inhibition of proteasomal degradation of target proteins increases the autophagy by the compensatory activation. Unfolded or misfolded proteins caused the ER stress, subsequently increasing the expression of ATG genes. In addition, the accumulation of unfolded or misfolded proteins stimulates the phosphorylation of p62 by ULK1. Phosphorylation of p62 positively modulates the binding of p62 to polyubiquitinated proteins and the recruitment of autophagy machinery to the target protein. Deficiency of proteasome components or the suppression of its activity was known to induce the transcriptional and translational expression of p62 and the p62 protein through the activation of transcription factor Nrf2. In summary, Nrf2 increases the transcription of p62, and the increased p62 binds to and stabilizes Nrf2, which then continually regulates the levels of p62 and Nrf2 through the positive feedback mechanism. 
or inactivated proteasomes and their subunits are degraded by selective autophagy, where p62 recognizes the polyubiquitin chains of targets formed on the proteasome surface and induces their degradation (66). Removal of these inactive proteasomes plays an important role in maintaining a constant pool of normal proteasomes in the cell. If the inactive proteasome is not properly removed because of the inhibition of autophagy, the accumulation of inactive proteasome will be resulted, which would then reduce the removal of ubiquitinated proteins through the UPS. Thus, the inhibition of autophagy reduces the UPS-mediated degradation of the ubiquitinated proteins through various p62-related pathways (Fig. 2).

In contrast, the inhibition of proteasomal degradation of ubiquitinated proteins causes the increase of autophagy, in which p62 play a role. Increase of intracellularly unfolded or misfolded proteins because of the proteasome inhibition triggers the phosphorylation of p62 at S405 (equivalent to S403 in humans) and S409 by ULK1. This modification of p62 stimulates its ability to directly bind to the polyubiquitinated target proteins (67). In particular, the phosphorylation of p62 at the S409 residue is essential for the recruitment of autophagy machinery to the targets, promoting the phosphorylation of p62-S405 by ULK1 and CK2 (68).

In addition, the deficiency of proteasome components increases the transcription and protein synthesis of $p 62$ by increasing the transcription of the Nrf2 protein (57). Nrf2 is a transcription factor that regulates the expression of several anti-oxidant genes and is degraded by the proteasome in a normal condition. On the other hand, Nrf2 increases the transcription of p62 under stress, and the increased p62 binds to Nrf2 and stabilizes Nrf2, which regulates the levels of p62 and Nrf2, continually, through the positive feedback mechanism $(69,70)$.

\section{THE CENTRAL ROLE OF p62 IN MITOPHAGY AND THE PROGRESSION OF PARKINSON'S DISEASE}

Parkinson's disease (PD) is one of the most common neurodegenerative diseases; about $2 \%$ of the population over age 65 is affected. The causes of PD are diverse and not yet clearly elucidated, but mutations in numerous genes, including PINK1 and parkin, are known to be closely associated with the development of familial forms of PD. The Ser/Thr protein kinase PINK1 and ubiquitin E3 ligase parkin induce mitophagy through a process known as the PINK1-parkin pathway, promoting mitochondrial quality control $(71,72)$. In mitophagy, parkin ubiquitinates the mitochondrial outer membrane proteins, and p62 recognizes these ubiquitinated proteins and initiates the formation of autophagosomes by recruiting LC3. Phosphorylation of parkin by PINK1 also promotes the binding of p62 to LC3 via the LIR domain. Involved in the formation of autophagosomes, p62 acts as a key component of the PINK1-parkin pathway. In a recent study, parkin was shown to bind directly to and to ubiquitinate p62, promoting its proteasomal degradation (73). These data suggested that parkin, a key component of the PINK1-parkin pathway, and p62, a key component for eliminating intracellular toxic protein aggregates, are closely linked functionally. Moreover, a defective function of 62 or/and its altered modulation somehow contributes to the pathogenesis of PD via the action of parkin.

In addition, p62 was commonly detected in the ubiquitinated protein aggregates of Lewy bodies from PD patients (74). Since p62 plays a role in the degradation of misfolded or unfolded proteins, dysregulation of the p62 function in CNS leads to an increase of the protein aggregates, consequently causing the neurodegenerative pathology. A defect in proper p62 function was also associated with increased aggregate formation of $\alpha$-synuclein, with a resultant toxic effect. For example, rotenone treatment increased the aggregation of $\alpha$-synuclein in the substantia nigra and degradation of dopaminergic neurons, coupled with increased expression of p62 (75). It is also known that p62 plays an important role in autophagic degradation of $\alpha$-synuclein inclusions. In this process, p62 binds to NBR1 and acts together as adaptor and cargo receptors for selective autophagy of ubiquitinated targets (33). As described previously, p62 was detected within the ubiquitinated protein aggregates of the Lewy bodies from PD patients. Increased protein aggregation resulted in increased expression of p62, which then stimulates the autophagy pathway to protect cells in a counteractive way. However, autophagic processes for the degradation of these aggregates did not work properly in PD. As a result, toxic protein aggregates containing $\alpha$-synuclein and p62 accumulate in the neuronal cells, causing the synucleopathy, cell death, and the progression of $\mathrm{PD}$, including cognitive deficits, and motor symptoms.

\section{CONCLUSION}

The UPS and autophagy were previously thought to be completely and independently operating and not related to each other. However, recent studies have shown a close link between them. For example, both systems employed ubiquitination on the target, and there are common modulators that operate on both systems. For example, USP14, known to inhibit protein degradation via the UPS by cleaving the K48-linked ubiquitin chain from the target, could also modulate the autophagy by promoting the K63-linked deubiquitination of specific targets, such as Beclin1. In addition to USP14, several other proteins, such as El24, p62, ATG16, and HDAC6, are known to regulate both the UPS and autophagy. In particular, p62 participates in many different cellular processes pertaining to protein homeostasis and plays an important role in regulating both activities between the UPS and autophagy. Furthermore, p62 interacts with the parkin to remove the protein aggregates as well as to stimulate the mitochondrial quality control, and alteration of this interaction 
or their regulation somehow contributes to the pathogenesis of Parkinson's disease. Despite the advances in understanding the molecular mechanism of the UPS and autophagy, there are still many questions to address about the detailed relationship and precise underlying control mechanisms between the two pathways under the specific cell context. Among the numerous questions, the crucial one is how to specifically find out whether the ubiquitinated target is degraded by either the UPS or autophagy, and how to control the relative activities of the UPS or/and autophagy. Because the UPS and autophagy play various important roles in cells and are linked to multiple diseases, understanding these complicated systems would contribute a novel but promising therapeutic development to these diseases.

\section{ACKNOWLEDGEMENTS}

This work was supported by grant from the National Research Foundation of Korea (NRF) (2018R1A2B2003955 to KCC) funded by the Ministry of Science, ICT \& Future Planning (MSIP), Republic of Korea, and by grant from the Korea Healthcare Technology R\&D Project (HI17C0936 to KCC) through the Korea Health Industry Development Institute (KHIDI), funded by the Ministry of Health \& Welfare, Republic of Korea.

\section{CONFLICTS OF INTEREST}

The authors have no conflicting interests.

\section{REFERENCES}

1. Komander D and Rape M (2012) The ubiquitin code. Annu Rev Biochem 81, 203-229

2. Klionsky DJ, Abdalla FC, Abeliovich $\mathrm{H}$ et al (2008) Guidelines for the use and interpretation of assays for monitoring autophagy in higher eukaryotes. Autophagy 4, 151-175

3. Ohtake F and Tsuchiya H (2017) The emerging complexity of ubiquitin architecture. J Biochem 161, 125-133

4. Schmidt M and Finley D (2014) Regulation of proteasome activity in health and disease. Biochim Biophys Acta $1843,13-25$

5. Swatek KN and Komander D (2016) Ubiquitin modifications. Cell Res 26, 399-422

6. Kwon YT and Ciechanover A (2017) The ubiquitin code in the ubiquitin-proteasome system and autophagy. Trends Biochem Sci 42, 873-886

7. Dikic I (2017) Proteasomal and autophagic degradation systems. Annu Rev Biochem 86, 193-224

8. Feng Y, He D, Yao Z and Klionsky DJ (2014) The machinery of macroautophagy. Cell Res 24, 24-41

9. Mizushima N, Kuma A, Kobayashi $Y$ et al (2003) Mouse Apg16L, a novel WD-repeat protein, targets to the autophagic isolation membrane with the Apg12-Apg5 conjugate. J Cell Sci 116, 1679-1688
10. Sarkar S (2013) Regulation of autophagy by mTORdependent and mTOR-independent pathways: autophagy dysfunction in neurodegenerative diseases and therapeutic application of autophagy enhancers. Biochem Soc Trans 41, 1103-1130

11. Moreau K, Renna M and Rubinsztein DC (2013) Connections between SNAREs and autophagy. Trends Biochem Sci 38, 57-63

12. Lilienbaum A (2013) Relationship between the proteasomal system and autophagy. Int J Biochem Mol Biol 4, 1-26

13. Tai HC and Schuman EM (2008) Ubiquitin, the proteasome and protein degradation in neuronal function and dysfunction. Nat Rev Neurosci 9, 826-838

14. Komatsu M, Waguri S, Koike M et al (2007) Homeostatic levels of p62 control cytoplasmic inclusion body formation in autophagy-deficient mice. Cell 131, 1149-1163

15. Labbadia J and Morimoto RI (2015) The biology of proteostasis in aging and disease. Annu Rev Biochem 84, 435-464

16. Yuan T, Yan F, Ying $M$ et al (2018) Inhibition of ubiquitin-specific proteases as a novel anticancer therapeutic strategy. Front Pharmacol 9, 1080

17. Russell RC, Yuan HX and Guan KL (2014) Autophagy regulation by nutrient signaling. Cell Res 24, 42-57

18. Korolchuk VI, Menzies FM and Rubinsztein DC (2009) A novel link between autophagy and the ubiquitinproteasome system. Autophagy 5, 862-863

19. Nam T, Han JH, Devkota S and Lee HW (2017) Emerging paradigm of crosstalk between autophagy and the ubiquitin-proteasome system. Mol Cells 40, 897-905

20. Xu D, Shan B, Sun H et al (2016) USP14 regulates autophagy by suppressing K63 ubiquitination of Beclin 1. Genes Dev 30, 1718-1730

21. Min Y, Lee S, Kim MJ, Chun E and Lee KY (2017) Ubiquitin-specific protease 14 negatively regulates toll-like receptor 4-mediated signaling and autophagy induction by inhibiting ubiquitination of TAK1-binding protein 2 and Beclin 1. Front Immunol 8, 1827

22. Kim E, Park S, Lee JH et al (2018) Dual function of USP14 deubiquitinase in cellular proteasomal activity and autophagic flux. Cell Rep 24, 732-743

23. Gu Z, Flemington $\mathrm{C}$, Chittenden $\mathrm{T}$ and Zambetti GP (2000) ei24, a p53 response gene involved in growth suppression and apoptosis. Mol Cell Biol 20, 233-241

24. Hwang M, Jun DW, Kang EH et al (2019) El24, as a component of autophagy, is involved in pancreatic cell proliferation. Front Oncol 9, 652

25. Tian Y, Li Z, Hu W et al (2010) C. elegans screen identifies autophagy genes specific to multicellular organisms. Cell 141, 1042-1055

26. Zhao $Y G$, Zhao $H$, Miao L, Wang L, Sun F and Zhang $H$ (2012) The p53-induced gene Ei24 is an essential component of the basal autophagy pathway. J Biol Chem 287, 42053-42063

27. Choi JM, Devkota S, Sung YH and Lee HW (2013) El24 regulates epithelial-to-mesenchymal transition and tumor progression by suppressing TRAF2-mediated NF- $\mathrm{KB}$ activity. Oncotarget 4, 2383-2396

28. Devkota S, Sung YH, Choi JM et al (2012) Ei24-deficiency attenuates protein kinase $\mathrm{C} \alpha$ signaling and skin 
carcinogenesis in mice. Int J Biochem Cell Biol 44, 1887-1896

29. Chen D, Gould C, Garza R, Gao T, Hampton RY and Newton AC (2007) Amplitude control of protein kinase C by RINCK, a novel E3 ubiquitin ligase. J Biol Chem 282, 33776-33787

30. Devkota S, Jeong $\mathrm{H}$, Kim $\mathrm{Y}$ et al (2016) Functional characterization of El24-induced autophagy in the degradation of RING-domain E3 ligases. Autophagy 12, 2038-2053

31. Hou B, Wang G, Gao Q et al (2019) SQSTM1/p62 loss reverses the inhibitory effect of sunitinib on autophagy independent of AMPK signaling. Sci Rep 9, 11087

32. Duran A, Amanchy R, Linares JF et al (2011) p62 is a key regulator of nutrient sensing in the mTORC1 pathway. Mol Cell 44, 134-146

33. Lamark T, Kirkin V, Dikic I and Johansen T (2009) NBR1 and p62 as cargo receptors for selective autophagy of ubiquitinated targets. Cell Cycle 8, 1986-1990

34. Matsumoto G, Wada K, Okuno M, Kurosawa $M$ and Nukina N (2011) Serine 403 phosphorylation of p62/SQSTM1 regulates selective autophagic clearance of ubiquitinated proteins. Mol Cell 44, 279-289

35. Kuma A, Mizushima N, Ishihara N and Ohsumi Y (2002) Formation of the approximately 350-kDa Apg12-Apg5·Apg16 multimeric complex, mediated by Apg 16 oligomerization, is essential for autophagy in yeast. J Biol Chem 277, 18619-18625

36. Fujita N, Saitoh T, Kageyama S, Akira S, Noda T and Yoshimori T (2009) Differential involvement of Atg16L1 in crohn disease and canonical autophagy analysis of the organization of the Atg16L1 complex in fibroblasts. J Biol Chem 284, 32602-32609

37. Xiong Q, Ünal C, Matthias J, Steinert $M$ and Eichinger L (2015) The phenotypes of ATG9, ATG16 and ATG9/16 knock-out mutants imply autophagy-dependent and -independent functions. Open Biol 5, 150008

38. Xiong Q, Fischer S, Karow M, Müller R, Meßling S and Eichinger L (2018) Atg16 mediates the autophagic degradation of the $19 \mathrm{~S}$ proteasomal subunits psmd 1 and psmd2. Eur J Cell Biol 97, 523-532

39. Lee JY, Koga H, Kawaguchi $Y$ et al (2010) HDAC6 controls autophagosome maturation essential for ubiquitin-selective quality-control autophagy. EMBO J 29, 969-980

40. Kawaguchi Y, Kovacs JJ, McLaurin A, Vance JM, Ito A and Yao TP (2003) The deacetylase HDAC6 regulates aggresome formation and cell viability in response to misfolded protein stress. Cell 115, 727-738

41. Fusco C, Micale L, Egorov M et al (2012) The E3-ubiquitin ligase TRIM50 interacts with HDAC6 and p62 and promotes the sequestration and clearance of ubiquitinated proteins into the aggresome. PLoS One 7, e40440

42. Yan J, Seibenhener ML, Calderilla-Barbosa L et al (2013) SQSTM1/p62 interacts with HDAC6 and regulates deacetylase activity. PLoS One 8, e76016

43. Pandey UB, Nie Z, Batlevi Y et al (2007) HDAC6 rescues neurodegeneration and provides an essential link between autophagy and the UPS. Nature 447, 859-863

44. Schubert U, Anton LC, Gibbs J, Norbury CC, Yewdell JW and Bennink JR (2000) Rapid degradation of a large fraction of newly synthesized proteins by proteasomes. Nature 404, 770-774

45. Kopito RR (2000) Aggresomes, inclusion bodies and protein aggregation. Trends Cell Biol 10, 524-530

46. Cyr DM, Hohfeld J and Patterson C (2002) Protein quality control: U-box-containing E3 ubiquitin ligases join the fold. Trends Biochem Sci 27, 368-375

47. Harada M, Hanada S, Toivola DM, Ghori N and Omary MB (2008) Autophagy activation by rapamycin eliminates mouse Mallory-Denk bodies and blocks their proteasome inhibitor-mediated formation. Hepatology 47, 2026-2035

48. Pan T, Kondo S, Zhu W, Xie W, Jankovic J and Le W (2008) Neuroprotection of rapamycin in lactacystininduced neurodegeneration via autophagy enhancement. Neurobiol Dis 32, 16-25

49. Cortes CJ and La Spada AR (2015) Autophagy in polyglutamine disease: imposing order on disorder or contributing to the chaos? Mol Cell Neurosci 66, 53-61

50. Hetz C, Chevet E and Oakes SA (2015). Proteostasis control by the unfolded protein response. Nat Cell Biol 17, 829-838

51. Munch D, Rodriguez E, Bressendorff S, Park OK, Hofius D and Petersen M (2014). Autophagy deficiency leads to accumulation of ubiquitinated proteins, ER stress and cell death in Arabidopsis. Autophagy 10, 1579-1587

52. Korolchuk VI, Mansilla A, Menzies FM and Rubinsztein DC (2009) Autophagy inhibition compromises degradation of ubiquitin-proteasome pathway substrates. Mol Cell 33, 517-527

53. Pankiv S, Clausen TH, Lamark T et al (2007) p62/SQSTM1 binds directly to Atg8/LC3 to facilitate degradation of ubiquitinated protein aggregates by autophagy. J Biol Chem 282, 24131-24145

54. Zaffagnini G and Martens S (2016) Mechanisms of selective autophagy. J Mol Biol 428, 1714-1724

55. Lin X, Li S, Zhao Y et al (2013) Interaction domains of p62: a bridge between p62 and selective autophagy. DNA Cell Biol 32, 220-227

56. Seibenhener ML, Babu JR, Geetha T, Wong HC, Krishna NR and Wooten MW (2004) Sequestosome 1/p62 is a polyubiquitin chain binding protein involved in ubiquitin proteasome degradation. Mol Cell Biol 24, 8055-8068

57. Puissant A, Fenouille $N$ and Auberger $P$ (2012) When autophagy meets cancer through p62/SQSTM1. Am J Cancer Res 2, 397-413

58. Thompson HG, Harris JW, Wold BJ, Lin F and Brody JP (2003) p62 overexpression in breast tumors and regulation by prostate-derived Ets factor in breast cancer cells. Oncogene 22, 2322-2333

59. Myeku N and Figueiredo-Pereira ME (2011) Dynamics of the degradation of ubiquitinated proteins by proteasomes and autophagy: association with sequestosome 1/p62. J Biol Chem 286, 22426-22440

60. Bjørkøy G, Lamark T, Brech A et al (2005) p62/SQSTM1 forms protein aggregates degraded by autophagy and has a protective effect on huntingtin-induced cell death. J Cell Biol 171, 603-614

61. Sahani MH, Itakura E and Mizushima N (2014) Expression of the autophagy substrate SQSTM1/p62 is restored during 
prolonged starvation depending on transcriptional upregulation and autophagy-derived amino acids. Autophagy 10, 431-441

62. Pankiv S, Lamark T, Bruun JA, Overvatn A, Bjorkoy G and Johansen $T$ (2010) Nucleocytoplasmic shuttling of p62/SQSTM1 and its role in recruitment of nuclear polyubiquitinated proteins to promyelocytic leukemia bodies. J Biol Chem 285, 5941-5953

63. Pikkarainen $\mathrm{M}$, Hartikainen $\mathrm{P}$, Soininen $\mathrm{H}$ and Alafuzoff I (2011) Distribution and pattern of pathology in subjects with familial or sporadic late-onset cerebellar ataxia as assessed by p62/sequestosome immunohistochemistry. Cerebellum 10, 720-731

64. Bayraktar O, Oral O, Kocaturk NM et al (2016) IBMPFD disease-causing mutant $\mathrm{VCP} / \mathrm{p} 97$ proteins are targets of autophagic-lysosomal degradation. PLoS One 11, e0164864

65. Marshall RS, McLoughlin F and Vierstra RD (2016) Autophagic turnover of inactive $26 \mathrm{~S}$ proteasomes in yeast is directed by the ubiquitin receptor Cue 5 and the Hsp42 chaperone. Cell Rep 6, 1717-1732

66. Cohen-Kaplan V, Ciechanover A and Livneh I (2017) Stress-induced polyubiquitination of proteasomal ubiquitin receptors targets the proteolytic complex for autophagic degradation. Autophagy 13, 759-760

67. Lim J, Lachenmayer ML, Wu S et al (2015) Proteotoxic stress induces phosphorylation of p62/SQSTM1 by ULK1 to regulate selective autophagic clearance of protein aggregates. PLoS Genet 11, e1004987

68. Moscat J, Diaz-Meco MT, Albert A and Campuzano S (2006) Cell signaling and function organized by PB1 domain interactions. Mol Cell 23, 631-640

69. Su H and Wang X (2011) p62 Stages an interplay between the ubiquitin-proteasome system and autophagy in the heart of defense against proteotoxic stress. Trends Cardiovasc Med 21, 224-228

70. Jain A, Lamark T, Sjottem E et al (2010) p62/SQSTM1 is a target gene for transcription factor NRF2 and creates a positive feedback loop by inducing antioxidant response element-driven gene transcription. J Biol Chem 285, 22576-22591

71. Deng H, Dodson MW, Huang H and Guo M (2008) The Parkinson's disease genes pink1 and parkin promote mitochondrial fission and/or inhibit fusion in Drosophila. Proc Natl Acad Sci U S A 105, 14503-14508

72. Yang Y, Gehrke S, Imai $Y$ et al (2006) Mitochondrial pathology and muscle and dopaminergic neuron degeneration caused inactivation of Drosophila Pink1 is rescued by Parkin. Proc Natl Acad Sci U S A 103, 10793-10798

73. Song P, Li S, Wu H et al (2016) Parkin promotes proteasomal degradation of p62: implication of selective vulnerability of neuronal cells in the pathogenesis of Parkinson's disease. Protein Cell 7, 114-129

74. Zatloukal K, Stumptner C, Fuchsbichler A et al (2002) p62 Is a common component of cytoplasmic inclusions in protein aggregation diseases. Am J Pathol 160, 255-263

75. Wu F, Xu HD, Guan JJ et al (2015) Rotenone impairs autophagic flux and lysosomal functions in Parkinson's disease. Neuroscience 284, 900-911 